\title{
El "BOоm" De la cIencia política en Chile: escuelas, MERCADO Y TENDENCIAS
}

\author{
Claudio Fuentes \\ Asociación Chilena de Ciencia Politica, Chile \\ Graciela Santana \\ Asociación Chilena de Ciencia Politica, Chile
}

\begin{abstract}
Resumen
La ciencia política en Chile ha estado tradicionalmente marcada por su eclecticismo teórico y la limitada cantidad de escuelas de formación. Sin embargo, a comienzos de esta década, se dio un giro relevante en la disciplina a partir de la apertura de un gran número de departamentos que imparten dicha carrera, particularmente a nivel de pregrado. En este artículo se da cuenta de este reciente desarrollo a partir del estudio analítico descriptivo de las mallas curriculares y de los perfiles de los profesionales que enseñan en dichas carreras. Se concluye que, aunque existe una importante transición disciplinaria con tendencias a la profesionalización, todavía se advierten un sinnúmero de precariedades en el ejercicio de la disciplina. Se advierte que gran parte del cambio se debe a las fuerzas del mercado y que es relevante una reflexión sobre qué tipo de profesionales se están formando y para qué.
\end{abstract}

\section{Abstract}

The development of political science in Chile has traditionally been defined by its eclecticism and the relatively small number of schools on this field. However, at the beginning of this decade, an important twist was taken after the opening of a great number of schools of political science in several universities within the country. This article describes this new development, studying the curricula and professional profiles of the professors who are teaching in such schools. The authors address that, even though it is possible to observe a relevant transition in the discipline in this country particularly concerning the professionalization of the field, there still several weaknesses to be solved. It is argued that market forces have driven such process and that it is crucial to think about the type of professionals are being instructed.

\section{PALABRAS CLAVE • Ciencia Política • Chile • Disciplina}

\section{INTRODUCCIÓN}

Desde su origen, la ciencia política ha sido una disciplina ecléctica que se ha nutrido de un sinnúmero de orientaciones teóricas y metodológicas incluyendo historia, filosofía, derecho, antropología y, más recientemente, sociología, administración pública, economía y estadística. La preocupación por ganar autonomía ha generado muchas veces ilusorios y áridos debates sobre la particularidad metodológica y/o teórica de esta disciplina por sobre otras. Esta discusión ha tenido importantes repercusiones en el tipo de formación y en la orientación de aquellos que se consideran cientistas políticos. 
El desarrollo del conductismo en la disciplina en la década de 1950 provocó una constante tensión entre los que buscan la "cientificidad" o el positivismo disciplinario (vía la adopción del método científico) en contra de aquellos que proclamaban la imposibilidad de aplicar criterios científicos a la realidad social. Entre medio, algunos estudiosos han intentado avanzar formulas mixtas que combinan la rigurosidad del método científico con la apertura hacia visiones más holísticas. Este debate nos ha acompañado desde el surgimiento de la disciplina y se ha revitalizado cuando autores como Gary King, Robert Keohane y Sidney Verba nos invitaron a adoptar un modelo o lógica de inferencia causal (1994) o cuando la propia Asociación de Ciencia Política de Estados Unidos se intentó revolucionar con la aparición de una Perestroika que cuestionó algunos de los supuestos empíricos dominantes en la disciplina. El debate sin duda promete continuar ${ }^{1}$ en cuanto cruza un aspecto no simplemente metodológico, sino que fundamentalmente teórico sobre las formas de concebir la realidad, la explicación de la causalidad, y la división entre sujeto y objeto, entre otros.

La existencia de 11 escuelas que otorgan el título o la mención en ciencia política a nivel de pregrado, 6 programas de maestría en ciencia política y 10 en gestión y administración pública llevan a pensar que la disciplina está de moda, cuestión interesante, pero al mismo tiempo preocupante. Inmediatamente surgen preguntas como: ¿Para dónde va la disciplina? ¿A qué velocidad avanzamos? ¿Con cuánta rigurosidad estamos moviéndonos? ¿Quién establece los parámetros para el desarrollo disciplinario? ¿Podemos y/ o debemos establecer estándares disciplinarios? ¿Cuál es el futuro laboral de aquellos egresados de ciencia política? ¿Requiere la sociedad chilena contar con una cantidad superior a cuatrocientos egresados al año? ¿Podrá absorber el mercado esta cantidad de estudiantes?

En este artículo se realiza una descripción analítica de la actual oferta académica en esta amplia disciplina que llamamos ciencia política en Chile. Se analizan las mallas curriculares y los perfiles de los profesionales que se desempeñan en los diversos programas en el pregrado. Así, este trabajo pretende contribuir a una reflexión sobre el estado de la disciplina, no analizando los principales nudos teóricos orientadores de ella, sino más bien a partir de una constatación empírica significativa: se está enseñando más ciencia política en nuestro país. Las preguntas que se pretenden responder en este artículo son ¿qué se está enseñando? y ¿quiénes lo están haciendo? A partir de allí, intentaremos derivar algunas hipótesis sobre el estado del arte y las necesidades urgentes en la disciplina.

\section{OFERTA EN CIENCIA POLÍTICA DE NIVEL PREGRADO}

A comienzos de esta década, se incrementó significativamente la oferta de ciencia política en Chile a nivel de pregrado (Tabla 1). En la actualidad, se están ofreciendo 11 carreras a nivel de pregrado que en su identificación aplican el término ciencia política. De ellas 6 licenciaturas ofrecen el título propiamente tal de cientista político, en dos casos se ofrece la licenciatura en ciencia política con el título de administración pública, dos carreras ofrecen ciencia política junto a otra materia como son relaciones internacionales y ciencia política y organizacional, y un caso en que se entrega ciencia política como mención. El promedio de estudiantes es de 50 por escuela, registrándose casos donde se superan los 100 estudiantes. Ninguna de las carreras se encuentra acreditada ante la Comisión Nacional de Acreditación de pregrado del ministerio de Educación. 
Dado que las condiciones del mercado laboral no han cambiado significativamente en los últimos 15 años, el crecimiento de la oferta parece tener que ver con la existencia de un sistema desregulado de apertura de carreras, el proceso de modernización del Estado que ha puesto un particular énfasis en temas de administración y gestión, el significativo incremento de profesores en la disciplina, y el relativo bajo costo que tiene la apertura de este tipo de carreras -las denominadas "carreras de pizarrón".

TABLA 1: Oferta en ciencia política en Chile (nivel pregrado)

\begin{tabular}{lllc}
\hline \multicolumn{1}{c}{ Universidad } & Grado & \multicolumn{1}{c}{ Título } & $\begin{array}{c}\text { Año } \\
\text { creación }\end{array}$ \\
\hline $\begin{array}{l}\text { Católica de Chile - Valparaíso } \\
\text { (PUC-Valpo) }\end{array}$ & Licenciatura & $\begin{array}{l}\text { Licenciado en Historia mención } \\
\text { Ciencia Política }\end{array}$ & 1976 \\
Concepción (UDEC) & Licenciatura & $\begin{array}{l}\text { Administrador Público mención } \\
\text { Ciencia Política }\end{array}$ & 1992 \\
Gabriela Mistral (UGM) & Licenciatura & Cientista político & 1993 \\
Católica de Chile (PUC) & Licenciatura & Cientista Político & 1995 \\
Del Desarrollo (UDD) & Licenciatura & Cientista Político & 2001 \\
Arcis (UA) & Licenciatura & Cientista Político & 2002 \\
Central (UCentral) & Licenciatura & Cientista Político & 2002 \\
Diego Portales (UDP) & Licenciatura & Cientista Político & 2002 \\
Acad. Humanismo Cristiano & Licenciatura & Cientista Político y Relaciones & 2003 \\
(UAHC) & & Internacionales & 2004 \\
Américas & & $\begin{array}{l}\text { Administrador en Gestión Pública y } \\
\text { Relaciones Internacionales }\end{array}$ & 2004 \\
Tecnológica metropolitana & Licenciatura & & \\
(UTEM) & & Cientista Político y Organizacional & 2004 \\
\hline & & & \\
\hline
\end{tabular}

A continuación presentamos una descripción de los cursos ofrecidos a partir de las mallas curriculares de las carreras de Ciencia Política en las universidades chilenas. Se recopiló la información de todas las universidades que hiciesen referencia a la disciplina, tanto como mención como título profesional, aun cuando alguna de ellas considere la administración pública dentro de su plan de estudios. A partir de las mallas curriculares se advierten nueve grandes temas que son abordadas con mayor o menor fuerza en los programas académicos. Ellos son:

- Administración Pública. Incluye aquellos ramos relacionados con los procesos de organización y gestión, tales como administración, gerencia pública, gestión, recursos humanos, logística, políticas empresariales, comportamiento organizacional.

- Economía. Asignaturas referentes a la economía y políticas públicas. 
- Análisis Político. Corresponden aquellas áreas ligadas principalmente al análisis politológico, tales como ciencia política, política comparada, análisis y coyuntura política, toma de decisiones políticas.

- Instituciones y Procesos. Aquellas materias que dicen relación con las estructuras y procedimientos políticos, dentro de este esquema se enmarca el derecho y los sistemas políticos.

- Defensa. Considera temas como relaciones cívico-militares, seguridad, defensa, geopolítica y resolución de conflictos.

- Relaciones Internacionales. La política exterior de los países, las organizaciones y estructuras internacionales, así como la historia internacional se reúnen dentro de esta clasificación.

- Teoría Política. La ética política, la filosofía y teoría política son fundamentales para conocer la base que sustenta la toma y aplicación de decisiones.

- Ciencias Sociales. Pese a no seguir en estricto rigor las disciplinas ligadas a las ciencias sociales, en este punto se ubican aquellos cursos complementarios al estudio politológico, como son antropología, comunicación política, epistemología, historia, ideas políticas, redacción y lenguaje, sociología y teología.

- Metodología. Se incluyen aquellas áreas de la metodología cualitativa y cuantitativa, así como la estadística, matemáticas y lógica.

A partir del acento temático, podríamos ordenar los distintos programas en tres grandes subgrupos: programas con una orientación hacia un perfil más politológico y con cursos disciplinarios tales como teoría, instituciones y procesos, relaciones internacionales, etc.; programas también con una orientación académica, pero con un énfasis marcado en las ciencias sociales en un sentido más amplio y cuyo perfil está más dominado por ramos como historia, y sociología; y programas orientados hacia la administración y gestión pública². La Tabla 2 sintetiza la cantidad de cursos ofrecidos por universidad de acuerdo a la descripción antes descrita. 
TABLA 2: Oferta curricular por universidad

\begin{tabular}{|c|c|c|c|c|c|c|c|c|c|c|c|}
\hline $\begin{array}{c}\text { Cursos } \\
\text { Mallas } \\
\text { Curriculares }\end{array}$ & 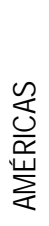 & $\frac{\mathscr{N}}{\underline{U}}$ & 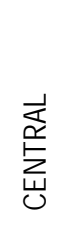 & $\begin{array}{l}\widehat{\mathbb{d}} \\
\stackrel{2}{0}\end{array}$ & 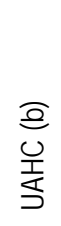 & $\begin{array}{l}\text { 0 } \\
0 \\
0\end{array}$ & 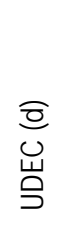 & $\begin{array}{l}\text { ब) } \\
\text { की }\end{array}$ & 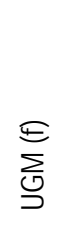 & $\begin{array}{l}\frac{0}{\sum_{1}} \\
5\end{array}$ & 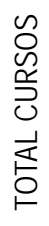 \\
\hline Administración Pública & 8 & - & 1 & - & - & 2 & 11 & 2 & - & 6 & 30 \\
\hline Análisis Político & 3 & 5 & 10 & 8 & 6 & 3 & 1 & 8 & 5 & 4 & 53 \\
\hline Ciencias Sociales & 3 & 4 & 5 & 4 & 11 & 7 & 4 & 7 & 15 & 5 & 65 \\
\hline Defensa & 1 & 3 & - & 1 & 3 & 1 & - & & & - & 9 \\
\hline Economía & 6 & 4 & 4 & 3 & 5 & 5 & 7 & 4 & 2 & 9 & 49 \\
\hline Instituciones y Procesos & 9 & 5 & 7 & 4 & 7 & 5 & 10 & 3 & 8 & 2 & 60 \\
\hline Metodología & 8 & 5 & 8 & 4 & 5 & 3 & 7 & 7 & 4 & 7 & 58 \\
\hline Relaciones Internacionales & 4 & 4 & 1 & 11 & 5 & 3 & 2 & 3 & 3 & - & 36 \\
\hline Teoría Política & - & 7 & 2 & 6 & 4 & 7 & - & 4 & 5 & 3 & 38 \\
\hline
\end{tabular}
(a) Pontificia Universidad Católica de Chile.
(b) Universidad Alberto Hurtado.
(c) Universidad del Desarrollo.
(d) Universidad de Concepción.
(e) Universidad Diego Portales.
(f) Universidad Gabriela Mistral.
(g) Universidad Técnica Metropolitana.

\section{PROGRAMAS DE ORIENTACIÓN POLITOLÓGICA}

Las universidades con una orientación politológica enfatizan temas vinculados a teoría política, instituciones y procesos, metodología y relaciones internacionales. Las casas de estudio superior que tienen tal finalidad son Arcis, Católica de Chile, Central, y Diego Portales.

En el caso de la Universidad Arcis, su programa tiene una mayor inclinación hacia la Ciencia Política, con un fuerte énfasis hacia la teoría política que es donde muestra más número de cursos, seguida por las áreas de metodología e instituciones y procesos, con igual cantidad de ramos. Lo anterior, está estrechamente relacionado con el perfil profesional propuesto por tal universidad, ya que definen tres líneas fundamentales: "una sólida formación teórica, una fuerte formación politológica y un riguroso desarrollo de la línea de investigación"³. Pese a que se busca formar un cientista político poli-funcional, colocan menos significado en el ámbito de la gestión pública, la administración y la gerencia pública. 
La licenciatura en ciencia política de la Pontificia Universidad Católica de Chile tiene como característica el que es un ciclo terminal, por lo que se accede a ella después de realizar un programa de bachillerato 0 haber cursado 200 créditos de una de las carreras consideradas compatibles por el Instituto de Ciencia Política. A partir de la admisión 2005 el plan de estudios ha sido modificado, ya que existe un total de 18 cursos mínimos equivalentes a 180 créditos, donde se establecen cursos básicos de cada ámbito, y 120 créditos correspondientes a cursos optativos, donde los alumnos pueden escoger la dirección de su preferencia.

La carrera pone un énfasis especial en los cursos vinculados al análisis politológico, las relaciones internacionales y la teoría política, situación que condice con el perfil presentado por la universidad, ya que se busca "formar licenciados capaces de analizar y evaluar los procesos políticos, tanto del gobierno interior como de las relaciones internacionales"4. Aunque en este programa se hace referencia que a través del plan de titulación se adquieren las destrezas para desenvolverse adecuadamente en el plano de las políticas públicas y las negociaciones políticas, son pocos los cursos que ofrece con esta orientación.

De las instituciones analizadas, la licenciatura en ciencia política de la Universidad Central, es la que ofrece mayor cantidad de cursos en el área politológica (10), con un enfoque de estudio de la disciplina desde la perspectiva de las instituciones y procesos, la metodología y la estadística. Cabe destacar las condiciones multidisciplinarias del plan de estudios, ya que los alumnos de licenciatura deben aprobar antes cuatro semestres, correspondientes a un bachillerato en ciencias políticas y administrativas ${ }^{5}$, donde reciben la misma formación que los estudiantes de administración pública, para luego optar por una de las dos carreras. El perfil profesional de la universidad está dirigido principalmente hacia el análisis político de los procesos de toma de decisiones.

La carrera de ciencia política en la Universidad Diego Portales, al igual que las licenciaturas analizadas anteriormente, tiene un fuerte componente de cursos politológicos dirigidos al análisis, hecho que se aprecia en el número de cursos de la formación principal y en los ramos de metodología y ciencias sociales que, por un lado, buscan entregar las herramientas que permitan una visión científica de la disciplina, a la vez que confiere los fundamentos teóricos en el área de las ciencias sociales, en el campo de la historia, derecho y sociología, para realizar las tareas demandadas. Asimismo, el perfil profesional proporcionado se relaciona con el plan de estudio, donde confluyen una educación polivalente y las competencias aplicadas.

\section{ORIENTACIÓN HACIA LAS CIENCIAS SOCIALES}

Un segundo grupo de universidades se orienta más hacia una formación también académica pero más centrada en las ciencias sociales, incluyendo historia, sociología y humanidades. Estas casas de estudio superior son la Universidad Gabriela Mistral, la Academia de Humanismo Cristiano y la Universidad del Desarrollo. 
La carrera de ciencia política de la Universidad Gabriela Mistral, al igual que en el caso de la PUC, cumple las condiciones de un ciclo terminal en unos casos, mientras que en otros se articula como complemento a otras carreras. Producto de lo anterior, su énfasis curricular (15 cursos) está puesto en cursos orientados a las ciencias sociales, con un número importante de asignaturas de historia y sociología, acompañado de un grupo menor de materias del ámbito de la ciencia política e instituciones y procesos. En este caso, la definición del perfil profesional queda diluida por cuanto se le considera en definitiva como un complemento en el marco de una doble titulación. Más que la búsqueda de una identidad dentro de la disciplina, se trata de una multiplicidad de conocimientos generales, lo que puede ser beneficioso en términos de la pluralidad de herramientas que suministra una carrera, pero que no se vincula mucho con el concepto academicista más tradicional de la formación politológica analizada en la sección anterior.

La licenciatura en ciencia política de la Universidad Academia de Humanismo Cristiano tiene una fuerte influencia de las ciencias sociales (11 materias) dentro de su plan de estudios, especialmente los cursos correspondientes a historia y sociología. Esta característica, la hacen una carrera multidisciplinaria y fuertemente centrada en las áreas mencionadas, con menor atención en la teoría política, las relaciones internacionales y los aspectos vinculados a las políticas públicas, aun cuando se advierte la búsqueda por seguir tres líneas fundamentales en su plan de estudios: relaciones internacionales, teoría del estado y economía.

La carrera de ciencia política y políticas públicas de la Universidad del Desarrollo comparte la mayoría de sus cursos entre las ciencias sociales y teoría política (7 ramos cada uno). Aun cuando la carrera se presenta como orientada hacia las políticas públicas, en la práctica las materias en este ámbito son sólo tres, lo que genera una contradicción con la mención o especialidad mencionada. Sin embargo, el perfil profesional mostrado es consecuente con la malla curricular propuesta. "Formar profesionales con capacidad de análisis, que sepan evaluar correctamente las necesidades de una sociedad dinámica y conozcan las herramientas para implementar cambios que mejoren la calidad de vida de las personas, dotarlos para todo ello de responsabilidad social, espíritu emprendedor, y sólida formación ética que les permitirá ser protagonistas activos de los desafíos del siglo XXI"6.

Finalmente, la Pontificia Universidad Católica de Valparaíso tiene un programa de licenciatura en Historia con mención en ciencia política. Éste tiene por objetivo formar licenciados en historia con una visión global en el ámbito de las humanidades, profundizando en el estudio de la disciplina histórica a la luz de las actuales investigaciones historiográficas y aportes de las ciencias sociales, en especial lo relacionado con la ciencia política y las relaciones internacionales. Dado que ciencia política es sólo una mención, tiene un fuerte acento en historia con cursos introductorios en ciencia política, educación cívica, relaciones internacionales y economía.

\section{ORIENTACIÓN HACIA LA ADMINISTRACIÓN PÚBLICA}

Aunque uno de los objetivos principales de este estudio es observar el estado de la educación de la ciencia política en el Chile de hoy, se han considerado estas carreras que, pese a que tienen la mayor cantidad de cursos en el área de administración pública, entregan el grado académico en

$6 \quad$ www.udd.cl/facultades/humanidades/licenpolitica_stgo 
ciencia política, situación que hace pertinente una revisión de qué se entiende en el país por enseñanza de la politología.

La carrera de ciencias políticas y administrativas de la Universidad de Concepción, tiene más de diez años de formación académica, con un marcado énfasis en los cursos de gerencia y administración pública e instituciones y procesos. Estos últimos conciernen en su mayoría a las materias de derecho, condición que puede ser explicada por la pertenencia a la facultad de Ciencias J urídicas y Sociales de esta universidad, lo que unido a una formación eminente en el área económica y estadística, busca configurar un profesional "que se identifica a plenitud con la problemática actual y futura de la Administración del Estado y en ese escenario, está en condiciones de satisfacer con calidad los requerimientos y demandas gerenciales y profesionales, de los Órganos de la Administración Pública del Estado"7.

En el caso de la Universidad de las Américas, la licenciatura en Ciencia Política y Administrativa está orientada fundamentalmente hacia la administración pública, por lo que un muy bajo porcentaje de cursos imparten materias de análisis político o de profundización en temas politológicos (política comparada, teoría, etc.). El plan de estudio, al igual que en el caso anterior, tiene una marcada inclinación hacia el área de instituciones y procesos en el área del derecho, además de la formación tradicional en gerencia pública, estadística y finanzas. Dicha instrucción es consecuente con ciertos aspectos del perfil profesional presentado, en términos del desempeño en instituciones del Estado en la planificación y ejecución de políticas públicas u otras áreas de la administración estatal.

Finalmente, la carrera de ciencia política y organizacional de la Universidad Tecnológica Metropolitana, es una licenciatura en administración pública, con una concentración fundamental en los temas de gestión y administración gubernamental, y con algunos ramos menores en ciencia política (4) y teoría política (3).

En términos generales, observamos que desde el punto de vista de mallas curriculares existen tres grandes tendencias en el desarrollo de lo que se denomina ciencia política. Una primera tendencia, se aproxima a un modelo que busca profundizar áreas de conocimientos propios de la politología, incluyendo instituciones y procesos, teoría, política comparada, relaciones internacionales y método. Una segunda aproximación, con un amplio número de cursos, observa la formación del cientista político como un subproducto del estudio multidisciplinario de lo que denominaríamos un "cientista social", con un fuerte acento en historia, teoría y sociología. Finalmente, un tercer núcleo de universidades profundiza lo relativo al gobierno y administración pública.

Asimismo, se observan algunas inconsistencias entre lo que es definido como el "perfil profesional" de las carreras y lo que efectivamente se está ofreciendo a los estudiantes a partir de un examen de las mallas curriculares. Por lo general, y dado que el mercado de trabajo está fuertemente vinculado con el Estado, la mayoría de las carreras ofrece un perfil profesional referente a la formación en análisis y gestión pública, cuestión que no se da en varios de los programas analizados pese a que en la descripción de la carrera se destaca este aspecto.

Un tema adicional se vincula con el futuro laboral del cientista político. En la actualidad no existen estadísticas que permitan dar cuenta del nivel de inserción laboral de los estudiantes luego de su 
egreso. Si se analiza con atención el Observatorio Futuro Laboral del Ministerio de Educación, de 113 carreras, la de ciencia política no es considerada en la muestra, aunque dos universidades chilenas tienen la licenciatura por más de cinco años. Asimismo, el Programa Servicio País de la Fundación Nacional para la Superación de la Pobreza, no solicita entre los profesionales requeridos cientistas políticos, aun cuando se advierte en la descripción de algunos cargos tareas propias de la disciplina.

Finalmente, las carreras estudiadas ofrecen muy pocas alternativas de profundización de un idioma extranjero, lo que aparece como fundamental a la hora de proyectar las posibilidades laborales, tanto en Chile como en el extranjero, de los politólogos.

\section{OFERTA A NIVEL DE POSTGRADO}

Coincidiendo con una tendencia generalizada a la adquisición de mayores calificaciones y educación de nivel superior en Chile, en la disciplina se da hoy una explosiva oferta de alternativas en el campo de ciencia política, relaciones internacionales, y política y gestión pública, entre otros.

La Tabla 3 muestra la oferta dividida por universidades nacionales y considera una división en tres grandes campos de acuerdo a la denominación que las propias casas de estudio asignan a sus programas de postgrado. A nivel de doctorado existen dos ofertas: una vinculada a estudios americanos y otra relacionada a Estudios Latinoamericanos y que se aproxima al campo de las Relaciones Internacionales. Ambas ofertas tienen un fuerte componente histórico y sociológico en su formación. A partir del 2006 el Instituto de Ciencia Política de la Universidad Católica abrirá un doctorado en ciencia política, el primero en Chile con características disciplinarias.

A nivel de magíster en ciencia política destacan los de la Pontificia Universidad Católica de Chile y los de la Universidad de Chile, siendo estos los más antiguos en el país. A ello se le suman hoy el programa de la Universidad Andrés Bello, la Universidad Arturo Prat, la Universidad Marítima y la Universidad Tecnológica Metropolitana.

La verdadera explosión de ofertas de postgrado se da en el campo de la política y gestión pública. En la actualidad se imparten 10 magíster de esa naturaleza y 7 diplomados vinculados a gestión. Existen, además, otros dos, uno en estudios de género y otro en filosofía política, a los que se agrega un diplomado en estudios estratégicos. La mayoría de estos programas son aplicados a la gestión pública y tienen por objetivo dotar a los estudiantes con herramientas metodológicas y teóricas para la práctica en el sector público. 
TABLA 3: Oferta a nivel de postgrado

\begin{tabular}{|c|c|c|c|}
\hline Universidad & $\begin{array}{l}\text { Ciencia Política } \\
\text { y Relaciones } \\
\text { Internacionales }\end{array}$ & Política y Gestión Pública & Otras áreas \\
\hline UAHC & & - MA Gerencia Pública & \\
\hline Adolfo Ibañez & & $\begin{array}{l}\text { - MA Gerencia y Políticas Públicas } \\
\text { - Dip. Alta Dirección Municipal }\end{array}$ & \\
\hline Alberto Hurtado & & - MA Gobierno y Sociedad & \\
\hline Andrés Bello & - MA & & \\
\hline Arcis & & & $\begin{array}{l}\text { - DOC Estudio } \\
\text { Latinoamericano }\end{array}$ \\
\hline \multirow[t]{2}{*}{ Arturo Prat } & - MA & - MA Gerencia Pública & \\
\hline & & - MA Políticas Públicas y Sociales & $\begin{array}{l}\text { - MA Estudios de } \\
\text { Género }\end{array}$ \\
\hline FLACSO-Chile & & $\begin{array}{l}\text { - MA Política y Gobierno(*) } \\
\text { - Dip. Gerencia Social }\end{array}$ & \\
\hline PUC & - MA & - Dip. Políticas Públicas & \\
\hline PUC-Valparaíso & & - MA Dirección Pública & \\
\hline U. Chile & $\begin{array}{l}\text { - MA CP } \\
\text { - MA RRII }\end{array}$ & $\begin{array}{l}\text { - MA Gobierno y Gerencia Pública } \\
\text { - MA Gestión y Política Pública } \\
\text { - Dip. Alta Dirección y Gobierno } \\
\text { Electrónico } \\
\text { - Dip. Género } \\
\text { - Dip. Metodología } \\
\text { - Dip. Diseño Política Pública en } \\
\text { Delincuencia }\end{array}$ & \\
\hline U. Marítima & $\begin{array}{l}\text { - MA } \\
\text { - Dip. }\end{array}$ & & $\begin{array}{l}\text { - Dip. Estudios } \\
\text { Estratégicos }\end{array}$ \\
\hline USACH & $\begin{array}{l}\text { - MA RRII } \\
\text { - MA Pol.Ex } \\
\text { - DOC Estudios } \\
\text { Americanos }\end{array}$ & & $\begin{array}{l}\text { - MA Filosofía } \\
\text { Política }\end{array}$ \\
\hline U. Talca & & - MA Gerencia y Gestión Pública & \\
\hline UTEM & - MA & & \\
\hline U. Viña del Mar & - Dip. RRII & & \\
\hline
\end{tabular}

${ }^{(*)}$ El caso del magíster de FLACSO-Chile, se realiza con la Universidad de Concepción. 


\section{EL PERFIL DE LOS PROFESORES DE PREGRADO}

Interesante resulta también conocer el perfil de los y las profesoras que imparten docencia en carreras vinculadas a ciencia política. Así, el estudio consideró que una descripción analítica del perfil profesional daría pistas sobre las principales tendencias presentes en el desarrollo disciplinario y las condiciones laborales de los profesionales. Cabe destacar que la información se solicitó a diez programas de pre grado, excluyendo el programa de la Pontificia Universidad Católica de Valparaíso por tratarse de una licenciatura en Historia con mención en ciencia política. Del universo de instituciones, sólo seis universidades facilitaron la información respecto de sus plantas de profesores en forma directa, proporcionando los datos académicos de sus docentes (Universidades Arcis, Católica de Chile, Academia de Humanismo Cristiano, Central, Concepción, y Diego Portales), mientras que dos lo hicieron de manera indirecta, a través de la información publicada en su sitio web. La Universidad de las Américas decidió no entregar los datos de sus académicos y la Universidad Tecnológica Metropolitana entregó información incompleta, por lo que no se consideraron en una muestra que alcanza a 147 académicos.

De la muestra se obtuvo información básica correspondiente al número de profesores por departamento, género, estudios de pregrado, estudios de postgrado (Master y Doctorado), país donde se desarrollaron los estudios y área de especialización de acuerdo a los grados académicos obtenidos en el postgrado. Además, en algunos casos se especificó la edad de los académicos y la jornada de trabajo. Cabe advertir que del total de académicos que imparten clases en los centros ya descritos, al menos nueve profesores dictan cursos en más de una universidad y tres de ellos en más de dos centros de ciencia política. Aquella tendencia parece aumentar a nivel de postgrado, aunque este trabajo no se detendrá en esa dimensión.

De los 147 profesionales, 44 tienen el grado de doctor (30\%), 27 son candidatos a doctor $(18,3 \%)$, 43 tienen el grado de magíster ${ }^{8}(29,3 \%)$, y 33 son profesionales o candidatos a magíster $(22,4 \%)$. Como más adelante observaremos, en los últimos cinco años se ha incrementado significativamente el número de doctores, aunque la tasa es todavía baja. Llama la atención el alto número de profesionales que son candidatos(as) a doctor y/ o magíster (25\%), lo que parece explicarse por las condiciones de reinserción de dichos profesionales en el país. Generalmente, se trata de personas que combinan la realización de sus tesis con docencia a fin de obtener su sustento.

Otra cifra relevante es que la disciplina está dominada por hombres, registrándose un muy bajo número de mujeres (24,5\%). Si bien Chile muestra bajos niveles de inserción laboral de la mujer (35 a 36\%), cuando se controló por la variable escolaridad, la inserción laboral de la mujer de alta escolaridad es cercana al $50 \%$, por lo que la cifra de un $23 \%$ se torna más dramática aún. Resulta de interés, sin embargo, constatar que el promedio de inserción laboral de la mujer en asuntos públicos a nivel nacional es cercano al 20\% (FLACSO-Chile 2005), lo que coincide con la cifra de profesoras impartiendo Ciencia Política.

Respecto de la formación de pregrado de los docentes, el $84 \%$ obtuvo su formación en Chile y la gran mayoría de ellos (88\%) lo hizo en una disciplina que no es ciencia política. Las disciplinas de formación básicas más recurrentes son historia (27,5\%), derecho (10,3\%), economía (8,3\%), so-

8 En el caso de los magíster se consideran aquellos que sólo tienen este grado, ya que la mayoría de los Doctores y Candidatos a Doctor cuentan con un grado de magíster. 
ciología $(9,7 \%)$ y filosofía $(9,7 \%)$. La relativa juventud de la disciplina explica que mientras los cientistas políticos que se educaron fuera del país tuvieron por formación de pregrado mayoritariamente la misma disciplina, en el caso de los que se formaron en Chile no fue el caso. Este hecho ciertamente redunda en el mayor eclecticismo disciplinario.

Al ordenar la obtención de grados por región geográfica, vemos que la gran mayoría de los magíster se obtienen en Chile, seguido de Estados Unidos y Europa. Otros países de América Latina no parecen ser atractivos para las personas que se forman en nuestro país. Esta tendencia geográfica se repite entre quienes están en proceso de titulación del magíster, donde todos los aspirantes desarrollan su especialización en Chile.

En cuanto a los doctorados un muy bajo porcentaje se realiza a nivel nacional, lo que se explica por una carencia de oferta, obteniendo un alto porcentaje su doctorado en Europa y principalmente en España $(68,2 \%)$. En el caso de los aspirantes a doctor se reitera la preferencia por las universidades europeas con el $59,2 \%$ de los candidatos.

TABLA 4: Lugar de obtención del grado académico

\begin{tabular}{lccccc}
\hline & $\begin{array}{c}\text { Chile } \\
\%\end{array}$ & $\begin{array}{c}\text { Europa } \\
\%\end{array}$ & $\begin{array}{c}\text { USA } \\
\%\end{array}$ & $\begin{array}{c}\text { A. Latina } \\
\%\end{array}$ & $\begin{array}{c}\text { Total } \\
\text { académicos }\end{array}$ \\
\hline Doctorado & 2,3 & 68,2 & 25,0 & 4,5 & 44 \\
Candidatos Doctor & 11,1 & 59,3 & 22,2 & 7,4 & 27 \\
Magíster & 62,8 & 14,0 & 16,3 & 7,0 & 43 \\
Candidatos Magíster & 10,0 & - & - & - & 10 \\
\hline
\end{tabular}

* En el caso de los magíster se consideran aquellos que sólo tienen este grado, ya que la mayoría de los Doctores y Candidatos a Doctor cuentan con un grado de magíster.

Respecto de la disciplina de especialización, en el caso de los magíster, el 37,2\% lo hace en ciencia política, mientras el resto lo realiza en otras áreas, incluyendo historia, filosofía, relaciones internacionales, políticas públicas, derecho, economía, geografía, ciencias sociales, trabajo social, y medio ambiente, entre otras. De esta misma forma, quienes están en proceso de obtención del magíster, marcan la preferencia por la especialización en ciencia política en el $90 \%$ de los casos.

Los doctorados, por su parte, en 25 casos se trata de títulos en ciencia política $(54,5 \%)$ y el resto incluye historia, filosofía, sociología, geografía, relaciones internacionales, economía y ciencias sociales. Siguiendo con esta tendencia, aunque en menor proporción, los candidatos a doctor eligen ciencia política como disciplina predominante $(44,4 \%)$, seguida por historia, filosofía y relaciones internacionales, entre otras. 
TABLA 5: Disciplina de especialización

\begin{tabular}{lccc}
\hline & $\begin{array}{c}\text { Ciencia Política } \\
\%\end{array}$ & $\begin{array}{c}\text { Otras disciplinas } \\
\%\end{array}$ & $\begin{array}{c}\text { Total } \\
\text { académicos }\end{array}$ \\
\hline Doctorado & 54,5 & 45,5 & 44 \\
Candidatos Doctor & 44,4 & 55,6 & 27 \\
Magíster & 37,2 & 62,8 & 43 \\
Candidatos Magíster & 90,0 & 10,0 & 10 \\
\hline
\end{tabular}

* En el caso de los magíster se consideran aquellos que sólo tienen este grado, pues la mayoría de los Doctores y Candidatos a Doctor cuentan con un grado de magíster.

Si consideramos la distinción de los grados de ciencia política (incluyendo relaciones internacionales) versus otras áreas de especialidad (historia, economía, sociología, antropología y filosofía) y lo cruzamos con el área geográfica, obtenemos que en el caso de los doctorados, la gran mayoría de los que hicieron su doctorado en ciencia política y relaciones internacionales lo obtuvieron en universidades europeas (15 casos), mientras que un reducido número lo hizo en universidades de Estados Unidos. La mayoría de estos últimos están enseñando en el Instituto de Ciencia Política de la Pontificia Universidad Católica de Chile, lo que marca en dicho instituto un sello muy distintivo en relación a otros centros donde predominan tradiciones europeas. En el caso de los magíster, casi todos los académicos cuyo grado es en ciencia política tienen una formación nacional y sólo en dos casos en EEUU.

Como se ha señalado, la totalidad de docentes candidatos a magíster lo realizan en Chile y a excepción de un caso, todos en Ciencia Política. Mientras que en los candidatos a doctorado, la tendencia de los doctorados se repite, ya que la mayoría realiza sus estudios en Ciencia Política, en Europa seguida de EEUU.

TABLA 6: Total académicos según grado y área geográfica de obtención título

\begin{tabular}{|c|c|c|c|c|c|}
\hline & Chile & Europa & USA & A. Latina & $\begin{array}{c}\text { Total } \\
\text { académicos }\end{array}$ \\
\hline Doctorado en Ciencia Política & 0 & 15 & 8 & 1 & \multirow{2}{*}{44} \\
\hline Doctorado en otra especialidad & 1 & 15 & 3 & 1 & \\
\hline Candidatos Doctor en Ciencia Política & 0 & 7 & 4 & 1 & 12 \\
\hline Candidatos Doctor en otra especialidad & 3 & 9 & 2 & 1 & 15 \\
\hline Magíster en Ciencia Política & 14 & - & 2 & - & \multirow[t]{2}{*}{43} \\
\hline Magíster en otra especialidad & 13 & 6 & 5 & 3 & \\
\hline Candidatos Magíster en Ciencia Política & 9 & - & - & - & 9 \\
\hline Candidatos Magíster en otra especialidad & 1 & - & - & - & 1 \\
\hline
\end{tabular}

* En el caso de los magíster se consideran aquellos que sólo tienen este grado, pues la mayoría de los Doctores y Candidatos a Doctor cuentan con un grado de magíster. 
Otra importante dimensión es la cantidad de profesionales con grados académicos por universidad. En el caso de los doctorados el mayor porcentaje de académicos con doctorado en ciencia política trabaja en la PUC (45,8\%), el $29 \%$ en la Universidad Central y el $16,6 \%$ en la UDP ${ }^{9}$. En el caso de los master, las dos universidades que concentran el mayor porcentaje de docentes con magíster en ciencia política son la Universidad Central con el 43,8\% y la Universidad del Desarrollo con el $31,3 \%$. 


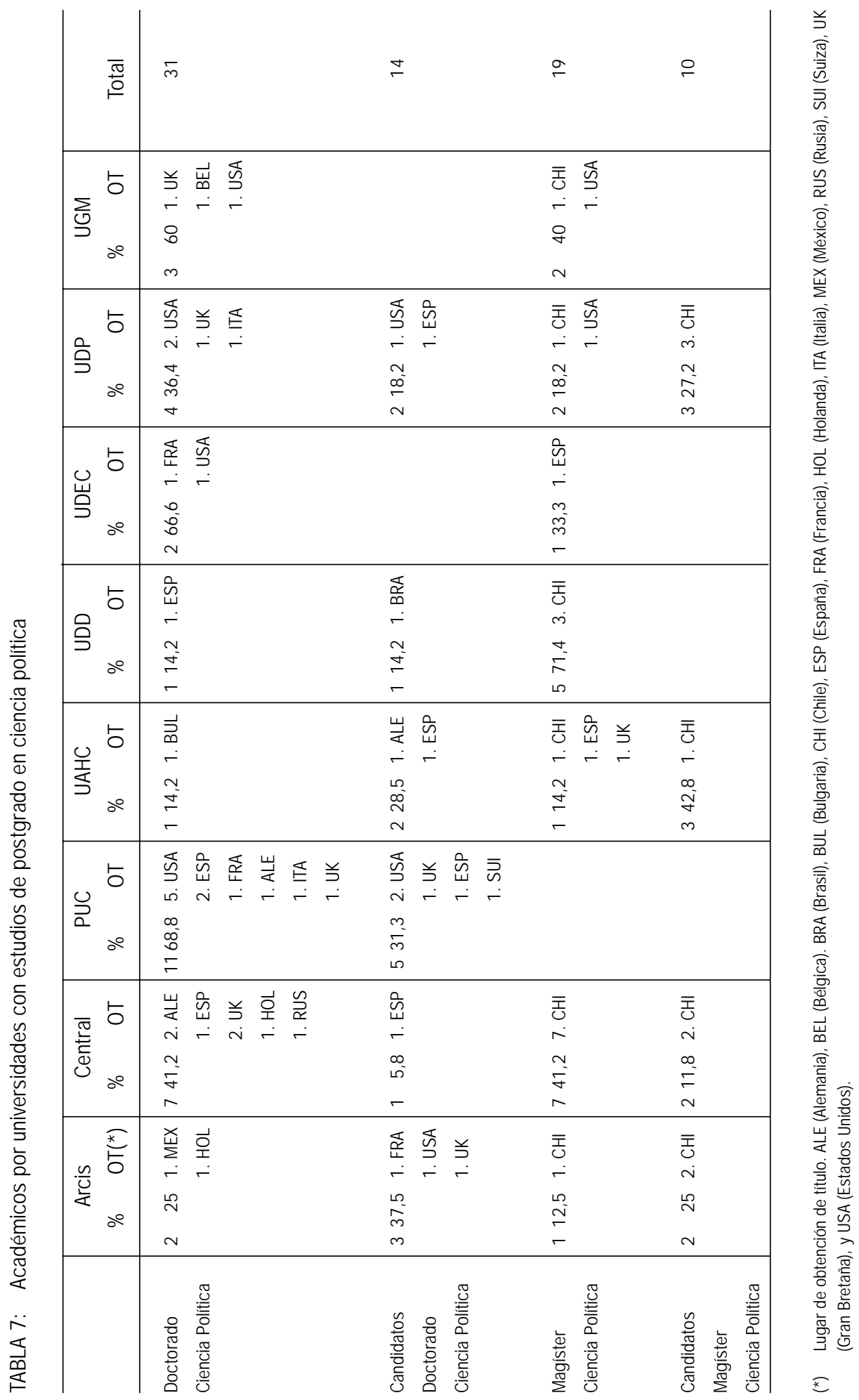


Al segmentar por zona geográfica los estudios de postgrado en ciencia política, de los académicos que enseñan esta disciplina en Chile, decíamos que la mayoría de quienes tienen un master en el área lo han cursado en el país, y lo han realizado preferentemente en la Universidad de Chile con el $76,9 \%$ y en la PUC con el $23 \%$. En el caso de los doctorados, no se aprecia una tendencia por alguna universidad en particular, aun cuando existe, como se ha señalado, una marcada preferencia por las universidades europeas de España, Inglaterra, Francia y Alemania. La Universidad Complutense de Madrid acapara el 13,3\% de las opciones. En el caso de los doctores en Estados Unidos, de ocho casos, tres corresponden a la Universidad de Notre Dame.

\section{DEDICACIÓN DE PROFESORES Y RANGO SALARIAL}

La obtención de datos se hizo más difícil cuando se trató de especificar la dedicación de profesores y los rangos y criterios para pago de sueldos por parte de las universidades. Lo anterior dice mucho sobre el predominio de una "cultura del secreto" en los centros universitarios en relación a temas donde podrían manifestarse algunas vulnerabilidades.

Pese a que se le consultó a diez universidades sobre la cantidad de profesores de tiempo completo, media jornada y profesores/hora, sólo seis departamentos entregaron la información completa (Tabla 8). 


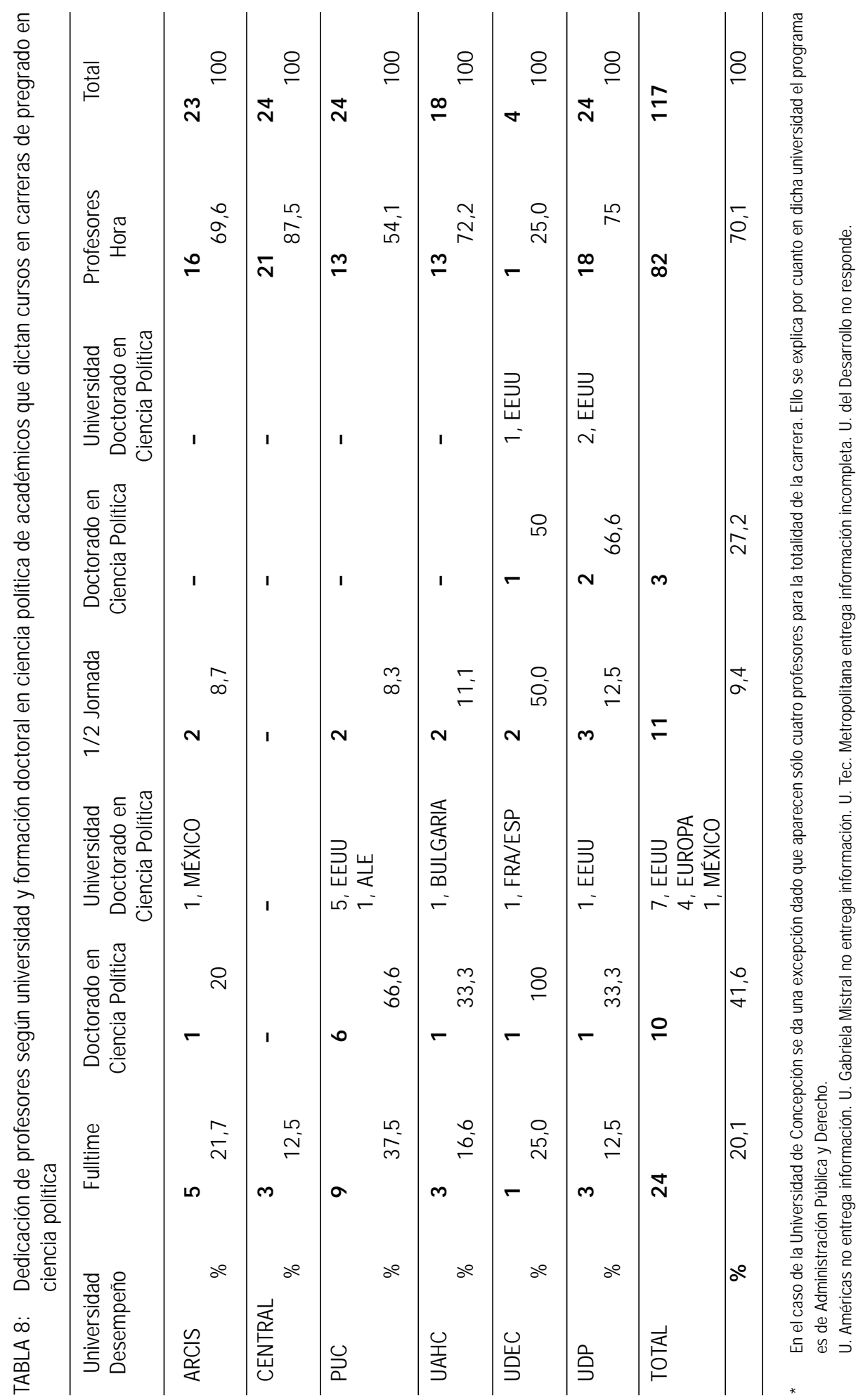


Excluyendo el programa de la Universidad de Concepción, el promedio de profesores por departamento es de 22,6 por facultad. La Pontificia Universidad Católica de Chile, la Universidad Central y la Universidad Diego Portales aparecen con igual número de académicos. No obstante, en este estudio no se obtuvo la cantidad total de estudiantes en cada carrera, lo que podría ser importante a la hora de evaluar el número de profesores.

La cantidad de profesores contratados en tiempo completo es un indicador relevante de la dedicación del programa a sus alumnos y de la posibilidad de los profesionales de realizar una carrera académica dentro de dichas instituciones. En ese sentido, la Pontificia Universidad Católica de Chile sobresale en relación al resto. No obstante, en la mayoría de los centros se advierte un alto nivel de contratación de profesores hora: 87,5\% en la Universidad Central, 72,2\% en la Academia de Humanismo Cristiano, 70,08\% en la Universidad Diego Portales.

En relación a los ingresos de los profesionales que se desempeñan en la disciplina, la gran mayoría de los Institutos no entregaron o no respondieron a la solicitud de información. A partir de informantes claves, se pudo constatar que los rangos de sueldo dependen mucho del grado académico, experiencia y del propio mercado (necesidad o no de un especialista en una materia). En muy pocos centros existen escalas de sueldos reguladas para la determinación de dichos ingresos. De la información recolectada en algunos centros se obtuvo que el rango de un profesional tiempo completo en una universidad oscila entre 1.300 y 3.400 dólares mensuales. En tanto, para profesores contratados por hora, el rango es de 300 a 500 dólares mensuales por un curso.

Otro aspecto relevante de ser analizado es la existencia de políticas de incentivos para los profesionales. Del conjunto de universidades donde se preguntó sobre este tipo de políticas a nivel de pregrado, en dos casos - Pontificia Universidad Católica de Chile y Diego Portales- se indicó que tales políticas eran aplicadas. En el primer caso, y por la estructura de la misma, existe una jerarquía académica bien establecida y donde los pasos para el ascenso en la carrera académica están establecidos. Adicionalmente, se paga una asignación por desempeño después de una evaluación que se realiza cada dos años. Además, los profesores reciben un incentivo por presentar proyectos de investigación y existe un pago adicional si dichos proyectos fueran aprobados. Existe un premio a la excelencia por publicaciones ISI y otorgamiento de sabáticos a los profesores adjuntos y titulares de seis meses cada seis años. Finalmente, la universidad también paga un incentivo por exclusividad a los profesores titulares ${ }^{10}$.

En el caso de la Universidad Diego Portales todavía no se ha desarrollado una política académica, aunque existen incentivos para la presentación de proyectos y se estimula la publicación en revistas con arbitraje internacional.

\section{INVESTIGACIÓN EN CIENCIA POLÍTICA}

Las condiciones para la investigación en Chile se ven afectadas por la estructura de inserción laboral de los profesionales, la carencia de recursos para hacer investigación y la orientación docente de los centros universitarios. De acuerdo a informantes claves en algunos de los centros de formación abordados en este trabajo, la carga de cursos para los profesores de tiempo com- 
pleto oscila entre dos y tres cursos por semestre. Aunque no existe información específica sobre la materia y menos en los casos de profesores de jornada parcial, es común la práctica de impartir cursos de pre y postgrado en tres, cuatro y hasta en seis centros de estudio diferentes, combinando clases a nivel de pre y postgrado en distintas universidades. Lo anterior repercute directamente en la posibilidad de desarrollar una producción de investigación sistemática en el campo de la ciencia política.

No existe una sistematización comprehensiva respecto del desarrollo de investigaciones y los recursos involucrados en este campo por institución académica. A modo de referencia, y con el objetivo de evaluar el nivel de productividad de las instituciones académicas, podemos observar las cifras de los proyectos obtenidos en concursos del Fondo Nacional de Ciencia y Tecnología (FONDECYT) desde 1985 a 2005. Se advierte que la mayoría de ellos son ganados por las dos principales universidades del país (de Chile y Católica de Chile), instituciones que tienen la mayor cantidad de profesores tiempo completo en sus respectivos institutos. En tercer lugar aparece FLACSO-Chile que tradicionalmente ha estado orientada a la investigación aplicada. La capacidad de desarrollar investigaciones está en directa relación con las condiciones laborales y dedicación de exclusividad de un profesional en algún centro universitario o de investigación.

TABLA 9: Proyectos FONDECYT ganados por centro académico en el área de ciencia política y legislación (1985-2005)

\begin{tabular}{lcccc}
\hline Centro & $\begin{array}{c}\text { Número de } \\
\text { proyectos }\end{array}$ & Porcentaje & $\begin{array}{c}\text { Recursos } \\
\text { Asignados } \\
\text { Miles \$ }\end{array}$ & Porcentaje \\
\hline Universidad de Chile & 24 & 31,2 & 206.779 & 28,3 \\
Universidad Católica de Chile & 19 & 24,7 & 191.316 & 26,2 \\
FLACSO-Chile & 8 & 10,4 & 41.591 & 5,7 \\
Universidad de Santiago de Chile & 3 & 3,9 & 51.216 & 7,0 \\
Universidad Católica - Valpo. & 3 & 3,9 & 21.724 & 3,0 \\
Universidad Alberto Hurtado & 3 & 3,9 & 20.136 & 2,8 \\
Otras & 17 & 22,0 & 191.659 & 27,0 \\
\hline
\end{tabular}

Fuente: Base de datos FONDECYT.

\section{$X$. TENDENCIAS PROFESIONALIZANTES DE LA DISCIPLINA}

El análisis precedente habla de una disciplina, en primer término, con una fuerte explosión de oferta docente a nivel de pre y postgrado; en segundo lugar, de naturaleza ecléctica, es decir, una disciplina que se divide en torno a tres ejes formativos (politología, ciencias sociales, y administración pública); y en tercer lugar, con un cuerpo de profesores creciente y proveniente de un amplio abanico de formaciones y visiones. Se advierte una fuerte influencia europea en la formación de postgrado y una menor de Estados Unidos. Otros países de América Latina no son considerados por los estudiantes para realizar sus especializaciones. 
El explosivo aumento de la oferta de ciencia política a nivel de pre y postgrado ha abierto un campo importante para los cientistas políticos. Esta dinámica, acompañada de procesos similares ocurridos en Argentina, Ecuador, y más recientemente Perú, han incentivado una mayor profesionalización de la disciplina. Ello se advierte a partir de tres tendencias:

1. Reclutamiento de profesores. En años recientes algunas universidades (Pontificia Universidad Católica de Chile y Diego Portales) han iniciado procesos de selección de profesionales por concurso público. Esta dinámica ha atraído a un cuerpo de profesionales de diversos países del mundo -incluidos chilenos y chilenas- que cuentan con altas calificaciones y que están por terminar 0 que recién han finalizado sus doctorados. El programa de reclutamiento de profesionales del Instituto de Ciencia Política de la PUC ha sido el más dinámico en los últimos años. Entre el 2003 y 2005 se incorporaron cinco nuevos doctores en ciencia política y se espera ampliar la cantidad de profesores significativamente en los próximos dos años. La Universidad Diego Portales ha comenzado un proceso de reclutamiento de profesores/as contratando dos doctores en el año 2005.

2. Publicaciones. Asimismo, se han comenzando a establecer mayores estándares de calidad en revistas nacionales y que incluyen comités editoriales, sistemas de arbitraje desarrollados sólo en algunos casos, periodicidad en las entregas, y establecimiento de estándares y normas editoriales. Hoy en Chile se publican las revistas Política (Universidad de Chile), Revista de Ciencia Política (Pontificia Universidad Católica de Chile), Estudios Internacionales (Universidad de Chile), Estudios Públicos (Centro de Estudios Públicos), Fuerzas Armadas y Sociedad (FLACSO-Chile) y Enfoques (Universidad Central).

3. Criterios de evaluación del trabajo académico. La mayor competencia también está generando espacios para un incremento de los estándares de calidad. Los profesores enfrentan la necesidad de publicar en revistas ISI, lo que ciertamente impone una nueva lógica de trabajo institucional, dado que los académicos responden a un estándar de trabajo internacional más que local.

\section{CUESTIONES PENDIENTES DEL DESARROLLO DISCIPLINARIO}

Este análisis del "estado del arte" de la disciplina deja más preguntas que respuestas. Por ejemplo, sería interesante analizar en más detalle los niveles de publicaciones de los/as profesoras que se desenvuelven en Chile, el tipo de investigaciones y las orientaciones teóricas de los mismos. La carencia de una sistematización mayor sobre nuestra disciplina es, sin duda, un vacío que se pretende resolver a través de la Asociación Chilena de Ciencia Política. Entre los aspectos pendientes del desarrollo de la disciplina y que se desprenden a partir de este trabajo se cuentan:

1. Información y transparencia. Parece muy grave que algunos centros de formación simplemente se nieguen a entregar información básica sobre el perfil de los profesores, dedicación y rangos de ingresos, entre otros temas. Parece también grave que los postulantes en Chile no tomen una decisión sobre dónde postular a esta carrera basada en criterios objetivos y comparables. No existe, por ejemplo, un registro accesible de la cantidad total de estudiantes por programa; proporción de estudiantes por profesores de tiempo completo; cantidad de profesores de planta, académicos contratados por jornada parcial y por hora; grados y calificaciones de los programas; cantidad de libros por estudiante; número de horas de los profesores destinado a atención de alumnos; proyectos de investigación ganados por profesor; beneficios e incentivos salariales de 
los profesores por proyectos de investigación ganados; etc. Esta información podría ser muy relevante para establecer criterios más sistemáticos de los que aquí hemos esbozado respecto de la calidad de los centros de estudios en ciencia política. La Asociación Chilena de Ciencia Política hará circular una encuesta en los centros de formación para sistematizar y dar cuenta de esta información a los postulantes a las carreras de la disciplina.

2. Criterios de asignación de remuneraciones de profesores. Otro de los temas que llama la atención del desarrollo de la profesión en Chile es el poco conocimiento y, en algunas universidades, la falta de criterios para la asignación de remuneraciones en relación al grado académico, las capacidades, jerarquía y responsabilidades. A partir de consultas con informantes claves, en varios casos se observa una lógica de libre mercado donde el o la profesora establece una negociación personal de su sueldo con la universidad. En muy pocos casos existen criterios conocidos de pago a profesores. Varias universidades se muestran muy reticentes a brindar una información abierta sobre el tipo de jerarquía académica existente (años de servicio, niveles y cantidad de publicaciones, evaluación docente, etc). Esto no parece ser una particularidad de la disciplina, sino más bien parece responder al sistema universitario en su conjunto.

3. Consistencia de la oferta docente. Otro de los problemas que se observa es la existencia de inconsistencias entre la oferta que los programas hacen en sus presentaciones a los estudiantes y lo que realmente se les imparte a partir de las mallas curriculares. En algunos casos, se promete el desarrollo de ciertos contenidos y competencias que no son desarrollados en el programa docente, cuestión que sin duda afectará al futuro profesional que se está educando en dichas carreras.

4. Acreditación y evaluación. Lo anterior lleva a un problema presente en el sistema universitario nacional y que se vincula con la ausencia de criterios y mecanismos para la acreditación y evaluación de los programas docentes. Hoy tal acreditación es voluntaria, por lo que pocos centros tienen incentivos para suscribirse a tales requerimientos. Dado el surgimiento explosivo de la carrera de ciencia política, se requeriría discutir dichos criterios. Lo anterior es un problema serio, por cuanto el nivel de ingreso de estudiantes a la disciplina es creciente y los problemas se verán en cuatro ó cinco años más, cuando exista una sobreoferta de cientistas políticos en el mercado nacional.

Otra de las paradojas de estas carreras, es que en los casos en que existe evaluación, muchas veces se pondera con mucho más fuerza el número y la relevancia de las publicaciones, asignándole menor importancia a la actividad docente propiamente tal, lo que incide en la calidad del profesor.

5. Estándares internacionales de calidad. Un aspecto crucial para el éxito de estos programas se refiere a la necesidad de acoger estándares internacionales de calidad para el trabajo de investigación y docente. Dado que Chile está convirtiéndose en un centro de atracción en cuanto a la formación de pre y postgrado, una estrategia que a las universidades les convendría adoptar es la acreditación de programas a nivel internacional y el establecimiento de estándares nacionales que puedan ser observados por actores 0 instituciones independientes. Lo anterior también se aplica a las publicaciones vigentes. Sería de alta conveniencia que ellas adoptaran un modelo de referato nacional e internacional para mejorar su calidad y garantizar la profesionalización de las mismas.

6. Acción afirmativa. Asimismo, y dada la sub-representación de mujeres en la disciplina, convendría promover políticas de contratación que prestaran más atención al equilibrio de género. 
7. Asociatividad. Otro de los desafíos de los distintos centros que se están formando es la posibilidad de buscar asociaciones virtuosas entre un centro y otro. Aunque varias de las opciones que hoy se están ofreciendo en el mercado aparecen como competitivas, parece existir un espacio para el desarrollo de iniciativas de investigación y docencia cooperativa en la medida en que los nichos temáticos tienden a diferenciarse.

8. Mercado laboral del cientista político. Se requiere también abordar con seriedad la inserción laboral de los egresados de las carreras. Lo anterior, implica una discusión a fondo sobre: (a) hasta qué punto los programas diseñados responden a las demandas del mercado laboral, (b) hasta qué punto existe una especialización o nichos temáticos en las distintas escuelas, y (c) qué tipo de seguimiento se realizará sobre esta materia por parte de los centros.

9. Investigación. Aunque no existe una sistematización acabada de la cantidad de investigación que se realiza, es probable que los centros de estudios universitarios viabilicen la investigación a través de FONDECYT que otorga limitados recursos para la disciplina, las propias universidades que han creado o podrían crear fondos reducidos para investigación, la empresa privada que podría interesarse en estudios aplicados (encuestas, impacto de política, etc.), fundaciones y organizaciones internacionales (que en el caso de Chile entregan pocos recursos para tales fines) y el Estado. Es probable que se dé una combinación de alianzas público-privadas en el futuro. No obstante, aquello quedará fuertemente determinado por el tipo de institución que se pretenda desarrollar. Hasta hoy, las universidades han apostado principalmente a la docencia.

\section{CONCLUSIONES}

La ciencia política en Chile comienza a cobrar una renovada cara a partir de esta década y como sub-producto de la apertura de nuevas escuelas de ciencia política. Se ha incrementado la oferta a nivel de pre y postgrado y, al mismo tiempo, ha aumentado significativamente el número de profesores que están de algún modo vinculados a esta disciplina. El desarrollo ha sido más bien ecléctico y conviven tres etiquetas: el desarrollo de escuelas con un fuerte acento académicodisciplinario con énfasis en teoría, relaciones internacionales, instituciones y procesos y métodos; un segundo modelo más vinculado a las ciencias sociales y donde la historia y sociología política juegan un papel preponderante; $y$, finalmente, un tipo de escuela muy relacionada con la gestión y política pública.

Desde el punto de vista del perfil de los profesores, se reproducen patrones pre-existentes con una fuerte presencia de profesionales que se incorporan a este campo por "adopción" más que formación. Predominan influencias europeas más que estadounidenses, aunque esta última tiende a crecer en el nivel de doctorados significativamente en los últimos tres años. El Instituto de Ciencia Política de la Pontificia Universidad Católica de Chile es el centro que cuenta con mayor presencia de cientistas políticos con formación realizada en Estados Unidos. En los otros centros predominan formaciones profesionales realizadas en Europa.

En cuanto a la experiencia formativa-docente, se advierte en términos generales falta de información pública sobre las condiciones en las que se imparten los cursos, tanto respecto de la infraestructura como de las condiciones en las que se da la experiencia docente. 
Siguiendo la tendencia del sistema educativo nacional, predominan una alta precariedad profesional en el ejercicio de la profesión, dominio de las reglas del mercado en cuanto a la contratación y asignación de salarios profesionales, falta de transparencia en varios centros académicos sobre las condiciones materiales y docentes para el ejercicio de la carrera y, con las excepciones del caso, falta de conocimiento de los criterios de evaluación de los programas en ciencia política. La Asociación Chilena de Ciencia Política pretende continuar recolectando información para llenar este vacío y contribuir a un debate fundado sobre el desarrollo disciplinario en Chile y las condiciones materiales para el ejercicio de la profesión.

En cuanto a la formación de los profesores que imparten clases hoy, en Chile se da una experiencia ecléctica, cuestión que podría convertirse en una oportunidad de diálogo interinstitucional e interdisciplinario. En la ciencia política confluyen un sinnúmero de disciplinas y orientaciones teóricas provenientes del derecho, la administración pública, filosofía, sociología, historia y antropología, entre otros, lo que es de por sí interesante y desafiante. Resultaría de interés sistematizar la producción académica reciente para evaluar los paradigmas predominantes. A partir del análisis de los perfiles profesionales es muy probable que ellos se vinculen preferentemente al nuevo-institucionalismo y constructivismo, y en forma más marginal con aproximaciones como la teoría de acción racional.

Dadas así las cosas, es probable que en el futuro se repitan las clásicas disputas teórico-metodológicas que ya se han dado en el primer mundo. En primer término, el carácter científico de la ciencia política seguramente generará más de alguna polémica, aunque, por el desarrollo disciplinario nacional, este debate no pareciera ser tan crucial. Aproximaciones eclécticas que enfatizan la rigurosidad de la observación empírica, pero que al mismo tiempo no se deslumbran con análisis exclusivos de regresiones, son las que probablemente predominarán. Seguramente las líneas de trabajo futuras enfatizarán la necesidad de estudios rigurosos que busquen relaciones causales contextualmente 0 históricamente determinadas (North, 1990; Huneeus y Lanas, 2002).

La generación de grandes paradigmas explicativos tampoco parece ser una tendencia en nuestra disciplina en el futuro próximo. Más bien se opta por aproximaciones teóricas de "rango medio", que destacan las particularidades de un caso históricamente determinado, pero que a partir de allí se avanza hacia inferencias causales más generales.

Desde el punto de vista temático, se estaría dando una tendencia hacia la investigación aplicada. Más que el anhelo de construcción de teoría - la que es desarrollada en espacios reducidos-, pareciera observarse en la mayoría de las investigaciones un intento de medir efectos inmediatos a procesos políticos de relevancia. La tensión entre agendas de investigación aplicadas versus teóricas y escuelas de ciencia política orientadas a la gestión pública versus aquellas más orientadas al desarrollo académico propiamente tal, parece ser que continuará en los próximos años. Es el mismo dilema que observará un estudiante de ciencia política que al cabo de sus años de estudio se preguntará ¿para qué me sirve ser un cientista político? Si bien este dilema está presente en la mayoría de las carreras - dado que la carrera de ciencia política es nueva y combina una serie de "saberes", y dada la desregulación del mercado en materia de creación de carreras- gran parte de la responsabilidad de responder a esta pregunta reside en las propios centros de ciencia política que deben articular programas para responder esta interrogante esencial: ¿para qué sirve un politólogo? Se produce, entonces, una constante tensión entre la compresión de la disciplina desde su visión académico-teórica y las necesidades que impone el mercado a jóvenes que 
después de cuatro o cinco años querrán insertarse en la vida laboral. ¿Deben las carreras convertirse en una "caja de herramientas aplicadas" a fin de preparar a los jóvenes para insertarse en un mundo laboral competitivo, o deben, en cambio, proveer de una "caja de saberes teóricos"? De preferirse la primera opción, las escuelas politológicas deberían potenciar cursos en metodología, estadística, política pública, economía e idiomas. De optarse por la segunda alternativa, debieran profundizar los aspectos vinculados a la teoría y análisis comparado, entre otros.

Otra de las paradojas que se observa en el desarrollo disciplinario es que pese a que la economía, estadística y sociología han ejercido una fuerte influencia en la forma en que la ciencia política ha ido cobrando forma en muchos países, en Chile existe parece ser que las vinculaciones entre aquellas disciplinas tienden a ser tenues y conflictivas. Se requeriría poner más atención (teórica y empírica) a la vinculación entre economía y política; entre las ciencias económicas y la ciencia política.

Enfrentamos una triple transición: se ha producido un verdadero "boom" de la formación en ciencia política, generándose una transición generacional en la disciplina y un incremento en número de académicos con formación fuera de la esfera europea - particularmente estadounidense- . Al igual que en otras disciplinas, parece ser que el mercado ha guiado fuertemente esta explosión de cientistas políticos. Corresponde, entonces, preguntarse qué sentido queremos darle a este proceso, bajo qué parámetros actuaremos, y con qué objetivos. Como se observa en este artículo, entre los temas que es necesario abordar en forma relativamente urgente está el acceso a la información sobre las carreras y sus características, la calidad y pertinencia de los programas y sistemas de evaluación. Y es que una gran masa de estudiantes aguarda respuestas para este nuevo desarrollo.

\section{REFERENCIAS}

Brady, Henry. 2004. "Introduction". Symposium: Two Paths to a Science of Politics. PS-Perspectives on Politics 2 (2): 295-300. Fernández, María de los Ángeles. 2003. "La Ciencia Política y su historia: los precursores chilenos". Clase magistral dictada en el Acto de Inauguración del Séptimo Programa de Magíster en Ciencia Política Integrada, de la Academia de Guerra Naval y la Universidad Marítima de Chile.

Flacso-Chile. 2005. Agenda Democrática. Santiago: Flacso.

Granato Jim, y Scioli Frank. 2004. "Identities, Interests, and the Future of Political Science". Symposium: Two Paths to a Science of Politics. PS-Perspectives on Politics 2 (2):313- 323.

Huneeus, Carlos y María Paz Lanas. 2002. "Ciencia política e historia. Eduardo Cruz-Coke y el Estado de bienestar en Chile, 1937-1938". Historia 25: 151-186.

King, Gary, Robert O. Kehoane y Sidney Verba. 1994. Designing Social Inquiry Scientific Interference in Qualitative Research. Princeton: Princenton University Press.

North, Douglass. 1990. Institutions, Institutional Change, and Economic Performance. Cambridge: Cambridge University Press. Smith, Rogers. 2004. "Puzzles, Proverbs, and Omega Matrices: The Scientific and Social Significance of Empirical Implications of Theoretical Models (EITM)". Symposium: Two Paths to a Science of Politics. PS-Perspectives on Politics 2 (2): 301-312. Zamitiz, Héctor. 1999. “Origen y Desarrollo de la Ciencia Política: Temas y Problemas". Convergencia 20: 89-122.

Claudio Fuentes Saavedra es Ph.D. Universidad de Carolina del Norte, Chapel Hill. Actualmente es Director de FLACSOChile y Presidente de la Asociación Chilena de Ciencia Política.

(E-mail: direccion@flacso.cl)

Graciela Santana es Licenciada en ciencia política, Pontificia Universidad Católica de Chile y alumna de Magíster en Ciencia Política de la Pontificia Universidad Católica de Chile. En estos días se desempeña como asistente de la Asociación Chilena de Ciencia Política.

(E-mail: accpchile@yahoo.es ) 